\title{
Experimental verification of depolarization effects in bioelectrical impedance measurement
}

\author{
Xiaoyan Chen*, Xinqiang Lv and Meng Du \\ College of Electronic Information and Automation, Tianjin University of Science and Technology, \\ Tianjin 300222, China
}

\begin{abstract}
The electrode polarization effects on bioelectrical impedance measurement at low-frequency cannot be ignored. In this paper, the bioelectrical data of mice livers are measured to specify the polarization effects on the bio-impedance measurement data. We firstly introduce the measurement system and methodology. Using the depolarization method, the corrected results are obtained. Besides, the specific effects of electrode polarization on bio-impedance measurement results are investigated using comparative analysis of the previous and posterior correction results from dielectric spectroscopy, Cole-Cole plot, conductivity and spectroscopy of dissipation tangent. Experimental results show that electrode polarization has a significant influence on the characteristic parameters of mouse liver tissues. To be specific, we see a low-frequency limit resistance $R_{0}$ increase by $19.29 \%$, a reactance peak $X_{P}$ increase by $8.50 \%$, a low-frequency limit conductivity $K_{l}$ decrease by $17.65 \%$ and a dissipation peak tangent decrease by $160 \%$.
\end{abstract}

Keywords: Electrode polarization, depolarization correction, impedance measurement, low-frequency

\section{Introduction}

The impedance characteristics of biological tissues change significantly with frequencies, which contains abundant inherent biophysical information. With further research on bioelectrical impedance detection and the biological effect of electromagnetic, the study of impedance spectroscopy characteristics of biological tissue becomes more and more significant.

The research of dielectric spectroscopy of biological tissue originated from the late 19th century [1]. Cole brothers established the three elements equivalent circuit model and proposed the Cole-Cole theory, introducing the concept that the electrical impedance of biological tissue can be represented by an arc of the fourth quadrant [2]. Besides, Schwan made theoretical explanation of dielectric spectroscopy, that three different frequencies dispersion co-exist in biological tissues, namely, $\alpha$-dispersion, $\beta$-dispersion and $\gamma$-dispersion [3]. These theories played an important role in promoting research on dielectric spectroscopy of tissues and cells.

\footnotetext{
${ }^{*}$ Corresponding author: Xiaoyan Chen, College of Electronic Information and Automation, Tianjin University of Science and Technology, Tianjin 300222, China. Tel.: 13752113103; Fax: (86)22-60275497; E-mail: cxywxr@tust.edu.cn.
}

0959-2989/14/\$27.50 @ 2014 - IOS Press and the authors. 
Early researchers focused primarily on measuring resistance and capacitance of biological tissues. Hermann discovered that resistance is related to the direction of the applied current to the skeletal muscle when measuring the resistance of frog skeletal muscle [4]. Rigauld B measured the impedance characteristics of sheep skeletal muscles, livers, and other tissues in vitro with his colleagues, and drew the impedance spectra [5]. In China, researches on bioelectrical impedance are developing rapidly within these years. Lin measured the bioelectrical impedances in vitro, collecting a large variety of impedance parameters, and establishing a database of impedance spectra [6]. Ma investigated dielectric properties of human and rat blood and clarified the frequency characteristics of normal blood cell dielectric properties [7].

Although researches on the impedance spectroscopy characteristics of biological tissue have made outstanding progress, the effect of electrode polarization on the measurement system cannot be neglected. On the contacting surface of the electrode and the measured object, an electric dipole layer is formed and a jumping potential called electrode potential is generated. The electrode potential varies with the applied electrical signal. Resistance and capacitance induced by electrode polarization affected the tissues biological impedance spectroscopy measurement adversely, especially in the audio/radio frequency bands [8]. For cell suspensions, the effect of electrode polarization is strong enough to overshadow the useful bioelectrical information in the $\alpha$-dispersion, which makes it very difficult to distinguish the real and false information for diagnosis or treatment in clinical applications.

Over the past years, many methods have been proposed to solve the polarization problem. For instance, platinum is used as the electrode material for its surface can be covered with a layer of rough platinum black to further eliminate electrode polarization [9]. It is also reported that four-electrode array sensors are designed to measure the electrical parameters of tissues. However, these methods cannot completely eliminate electrode polarization and it inherently exists in the radio frequency regions. As a result, depolarization corrections are inevitably required.

In this paper, a depolarization correction method proposed by Raicu et al. [10] in 1998 is described, and newly designed experiments are carried out to test its performance. Finally, differences between parameters with and without corrections are obtained, which could be further used in clinical applications that require accurate dielectric spectroscopy.

\section{Methodology}

Raicu et al. proposed that the spurious impedance can be expressed as a combination of a series of frequency-dependent resistances $\left(R_{P}\right)$ and capacitances $\left(R_{P}\right)$. They are in series with the impedance of detected tissue represented by a parallel combination of resistance $R_{s}$ and capacitance $C_{s}$ of the measured tissue. The total impedance can be given as a function of the angular frequency

$$
Z(\omega)=R_{p}+\frac{R_{s}}{1+\left(\omega C_{s} R_{s}\right)^{2}}-j\left[\frac{1}{\omega C_{p}}+\frac{\omega C_{s} R_{s}^{2}}{1+\left(\omega C_{s} R_{s}\right)^{2}}\right]
$$

Where $\omega$ is the angular frequency $\omega=2 * p i * f, p i$ is the circumference ratio and $f$ is frequency, $j$ is an operator expressed square root of -1 .

Resistance and capacitance induced by polarization is given by Eq. (2). 


$$
R_{p}=A \omega^{-m}, C_{p}=B \omega^{-n}
$$

where $\mathrm{A}, \mathrm{B}, \mathrm{m}$ and $\mathrm{n}$ are positive constants. Assuming that both $C_{s}$ and $R_{s}$ are frequency-independent, the derivative of the real part of $Z(\omega)$ with respect to $\omega$ takes the following form:

$$
-\frac{d \operatorname{Re}[Z(\omega)]}{d \omega}=A m \omega^{-(1+m)}+\frac{2 \omega C_{s}^{2} R_{s}^{3}}{\left[1+\left(\omega C_{s} R_{s}\right)^{2}\right]^{2}}
$$

Likewise, the derivative of the imaginary part of $Z(\omega)$ with respect to $\omega$ takes the following form:

$$
\frac{d \operatorname{Im}[Z(\omega) / \omega]}{d \omega}=\frac{2-n}{B} \omega^{n-3}+\frac{2 \omega C_{s} R_{s}^{4}}{\left[1+\left(\omega C_{s} R_{s}\right)^{2}\right]^{2}}
$$

As $\omega \rightarrow 0$, the second term on the right-hand side of Eqs. (3) and (4) become negligible compared to the first term, thus we can obtain the approximated equations as Eq. (5).

$$
-\frac{d \operatorname{Re}[Z(\omega)]}{d \omega}=A m \omega^{-(1+m)}, \frac{d \operatorname{Im}[Z(\omega) / \omega]}{d \omega}=\frac{2-n}{B} \omega^{n-3}
$$

Take logarithm of the both sides of Eq. (5):

$$
\begin{aligned}
& \log \left\{-\frac{d \operatorname{Re}[Z(\omega)]}{d \omega}\right\}=-(1+m) \log \omega+\log (A m) \\
& \log \left\{\frac{d \operatorname{Im}[Z(\omega) / \omega]}{d \omega}\right\}=(n-3) \log \omega+\log \frac{2-n}{B}
\end{aligned}
$$

The real and imaginary part of $Z(\omega)$ corresponding to each frequency can be obtained from experimental data. When plotted against $\log \omega$, the curves are linear over the frequency range where electrode polarization dominates. By calculating the slope and intercept of the curves, constants $\mathrm{A}, \mathrm{B}$, $\mathrm{m}, \mathrm{n}$ can be determined and the spurious resistance and capacitances caused by electrode polarization can be obtained. Finally, depolarization correction can be accomplished through vector subtraction for each point of raw data. 


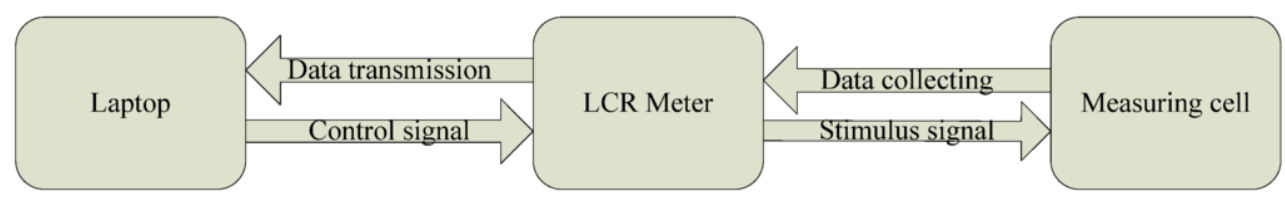

(a)

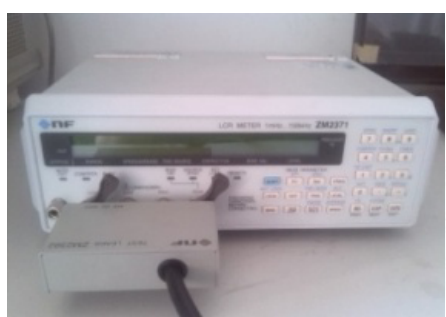

(b)

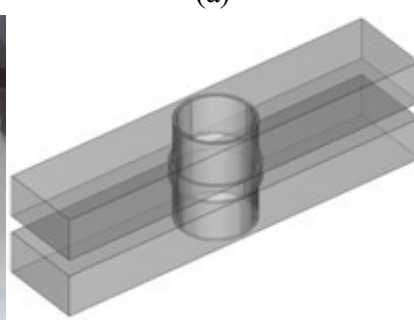

(c)

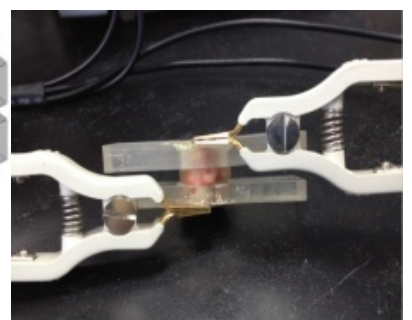

(d)

Fig. 1. Impedance measurement system. (a) Measurement system structure, (b) LCR Meter, (c) Measuring cellstructure and (d) Measuring cell.

Raicu et al. used this method to successfully correct their experimental results from rat liver in vivo and analyzed the effect of portal perfusion with isotonic glucose solution in 1998 [10]. Stoneman et al. also used this method to correct the data from normal and cancerous breast tissues in 2007, concluding that dielectric spectroscopy has the potential to become the feasible way to detect breast cancer in vivo [11].

\section{Experiments}

\subsection{Materials}

In order to verify the feasibility of the above method, an impedance measurement system (Figure 1) is built. It consists of a ZM2371 LCR meter from NF Company, a measurement cell, measurement electrodes and a PC laptop. 6 healthy female mice of the same batch from the Institute of Cancer Research (ICR), weighted ( $35 \pm 2) \mathrm{g}$, were prepared, dissected with their livers taken as tissues for test. Tested tissue is fully filled in the measurement cell after trimming and the cell is connected to a LCR meter through the adapter (ZM2392) and fixture before measurement. Room temperature is controlled at $(25 \pm 1)^{\circ} \mathrm{C}$.

\subsection{Measurement methods}

Firstly, we need to make sure LCR meter has been calibrated with open, short and load calibration before measurement. Then we put the trimmed samples into the measurement cell, set the LCR into $|Z|-\theta$ mode, and select 53 frequencies between $1-100 \mathrm{kHz}$ to measure the dielectric spectroscopy. The measured impedances and phases can be converted into the relative permittivity $\varepsilon_{r}$ and conductivity $K$ using the Eqs. (8)-(10):

$$
R=|Z| \cdot \cos \theta, X=|Z| \cdot \sin \theta, R_{s}=R-R_{p}, C_{s}=X-C_{p}
$$




$$
\begin{aligned}
& \varepsilon_{r}=C d / \varepsilon_{0} S, C=\frac{1}{2 \pi f} \cdot \frac{-C_{S}}{R_{S}^{2}+C_{S}{ }^{2}} \\
& K=d G / S, G=\frac{R_{s}}{R_{s}{ }^{2}+C_{S}{ }^{2}}
\end{aligned}
$$

where $C$ and $G$ are the measured sample equivalent capacitance and equivalent conductance, respectively. $d$ and $S$ are the sample length and the test chamber cross-sectional area. $f$ is the frequency and $\varepsilon_{0}=8.85419 \times 10^{-12} \mathrm{~F} / \mathrm{m}$ is the vacuum permittivity.

\section{Results and discussion}

\subsection{Parameter calculation}

The measured data against $\log \omega$ are plotted in Figure 2.

In Figure 2, the horizontal axis represents angular frequency and the vertical axis represents the left-hand side of Eqs. (6) and (7), respectively. Use the linear fitting function in Origin 8.5, two straight lines are obtained. Their slopes and intercepts can then be used to calculate the values of constants $A, B, m, n$ as: $A=4621.5, B=2.59 \mathrm{e}-4, m=0.21, n=0.68$.

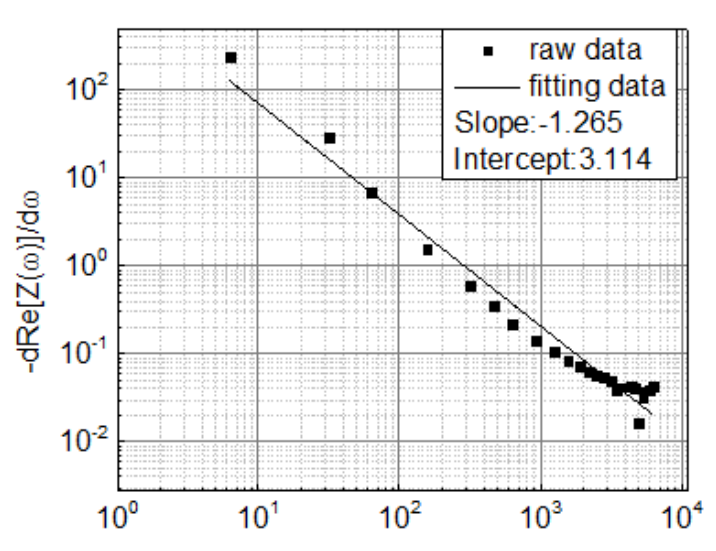

(a)

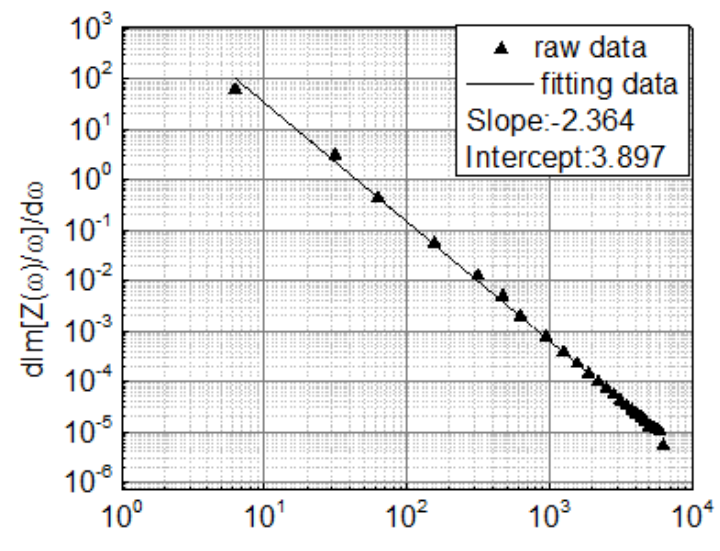

(b)

Fig. 2. Line fitting in depolarization correction. (a) Real part fitting and (b) Imaginary part fitting. 


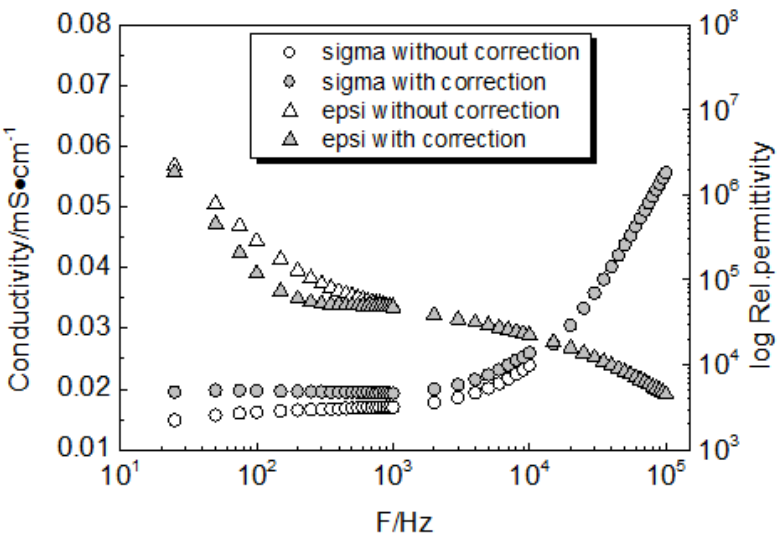

(a)

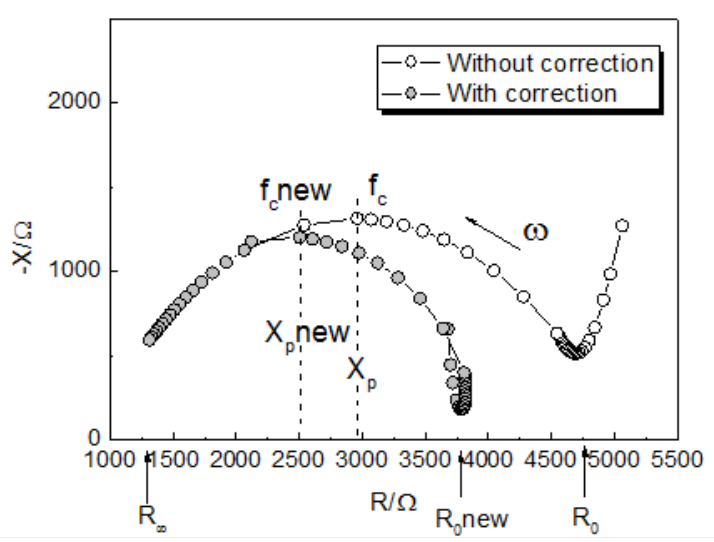

(b)

Fig. 3. Correction results in dielectric spectroscopy and Cole-Cole plot of impedance. (a) Dielectric spectroscopy of mouse liver and (b) Cole-Cole plot of impedance of mouse liver.

\subsection{Correction of the dielectric spectroscopy and Cole-Cole plot}

According to the results above, the resistance $R_{p}$ and capacitance $C_{p}$ induced by polarization can be obtained using Eq. (2). The conductivity and the permittivity calculated by Eqs. (8)-(10) at each frequency are plotted in Figure 3.

Figure 3(a) shows the dielectric spectroscopy of mouse liver tissues, the horizontal axis represents the excitation frequency and the vertical axis represents the conductivity and relative permittivity of tested samples. The curves indicate the nonlinear characteristics of conductivity and relative permittivity with frequency, which is accordance with the dispersion theory. Relative permittivity decreases and conductivity rises gradually with increasing frequency, which indicates that the dielectric properties of cell change from high resistance to low resistance. The main influence of electrode polarization on relative permittivity exists when frequency is below $1 \mathrm{kHz}$. While for conductivity, major impact exists when frequency is below $10 \mathrm{kHz}$. Electrode polarization increases the value of relative permittivity when frequency is below $1 \mathrm{kHz}$, while decreases the value of conductivity when frequency is below $10 \mathrm{kHz}$. After depolarization corrections, the effect of resistance and capacitance generated by electrode polarization can be eliminated.

Figure 3(b) shows the corrected results in Cole-Cole plot of tissue impedance. The vertical axis represents the minus reactance, and the horizontal axis represents the resistance with frequency gradually increasing from right to left. $R_{0}$ is corresponding to the minimum value of $X$ at the lowest frequency, and $R_{\infty}$ is corresponding to the minimum value of $X$ at infinite frequency. The peak value of $X$ corresponding to the top of the arc is marked as $X_{p}$ at $f_{c 1}$. By depolarization, it is obvious that the corrected data move to the direction of low impedance significantly at the low-frequency region compared with the raw measured data.

The corrected $R_{\text {Onew }}\left(3792.5 \Omega\right.$ ) reduces by $19.29 \%$ compared with the initial $R_{0}(4699.1 \Omega)$. And the corrected $X_{\text {pnew }}(1203.8 \Omega)$ reduces by $8.50 \%$ compared with the initial $X_{p}(1315.7 \Omega)$. Besides, there is no difference between $R_{\text {conew }}$ and $R_{\infty}, f_{\text {clnew }}$ and $f_{c 1}$, namely, $R_{\infty}=R_{\text {conew }}=1312.4 \Omega, f_{\text {clnew }}=f_{c 1}$ $=10 \mathrm{KHz}$. From Figure 3(b), the upturned phenomena at low frequency is improved obviously, which 
acts as a proof that the errors at low frequency are caused by the effects of electrode polarization, which can be eliminated using the depolarization method proposed in this paper.

\subsection{Correction of conductivity and the spectroscopy of dissipation tangent}

Figure 4(a) shows the corrected results in Cole-Cole plot of tissue conductivity. The horizontal axis represents the real part of the conductivity $\left(K^{\prime}=K\right)$, and the vertical axis represents the imaginary part of the conductivity $\left(K^{\prime \prime}=\left(\varepsilon-\varepsilon_{h}\right) \omega \varepsilon_{0}\right)$. The entire curve is an arc in the first quadrant, and frequency gradually increases from right to left. The value of $K^{\prime}$ corresponding to the minimum value of $K^{\prime \prime}$ at low and high frequency are $K_{l}$ and $K_{h}$, respectively. The peak value of $K^{\prime \prime}$ corresponding to the top of the arc is denoted as $K_{P}^{\prime \prime}$, and frequency corresponding to $K_{P}^{\prime \prime}$ is named as the characteristic frequency $f_{c 2}$. The low-frequency parts of the corrected data move to the direction of high conductivity significantly compared with the measured data. Corrected $K_{\text {lnew }}(0.02)$ rises by $17.65 \%$ compared with the initial $K_{l}(0.017)$. Other parameters don't change too much after corrections, for example, $K_{\text {hnew }}$ $=K_{h}=0.055, \quad f_{\text {c2new }}=f_{c 2}=25 \mathrm{KHz}, \quad K_{\text {Pnew }}^{\prime \prime}=K_{P}^{\prime \prime}=0.013$.

Figure 4(b) shows the correction results in the spectroscopy of dissipation tangent $\left(\tan \delta=\varepsilon^{\prime \prime} / \varepsilon^{\prime}\right)$. The horizontal axis represents frequency and the vertical axis represents the dissipation tangent. The entire curve indicates that the dissipation tangent of tissue changes with the frequency. Dissipation $\delta$ represents the ratio of cell loss energy to its stored energy. The most obvious difference between the two curves is the height of peak, and the difference firstly increases and then gradually reduces with increasing frequency. Corrected $\tan \delta_{\text {pnew }}(19.16)$ is 1.6 times larger than the initial $\tan \delta_{p}(7.37)$, and the two peak frequencies are also slightly different. These indicate electrodes polarization makes dissipation tangent of tissue reduce about 1.6 times than the actual value, and increases the peak frequency slightly.

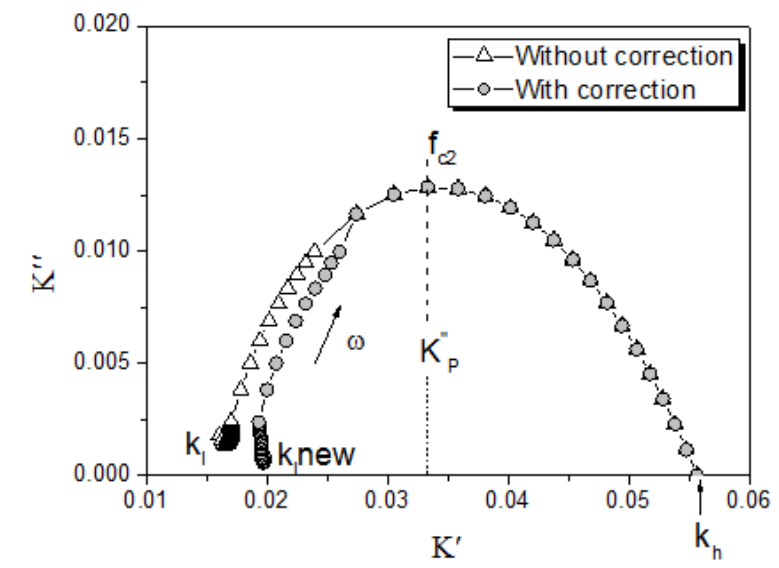

(a)

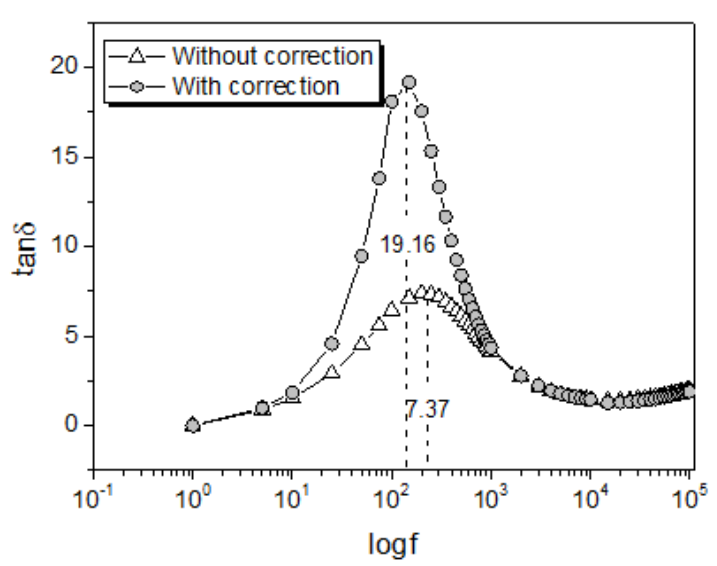

(b)

Fig. 4. Correction of conductivity and the spectroscopy of dissipation tangent. (a) Cole-Cole plot of conductivity of mouse liver and (b) The spectroscopy of dissipation tangent. 


\section{Conclusion}

To summarize, this paper presents a method to eliminate the effects of electrodes polarization, which can be used to correct experimental data (depolarization). Besides, the specific effects of electrode polarization in bio-impedance measurement results are investigated by comparative analysis of previous and posterior correction results. Electrode polarization effects have a significant influence on some characteristic parameters of mouse liver tissues: electrode polarization effects lead to a low-frequency limit resistance $R_{0}$ increase by $19.29 \%$, a reactance peak $X_{P}$ increase by $8.50 \%$, a low-frequency limit conductivity $K_{l}$ decrease by $17.65 \%$, and a dissipation peak tangent decrease by $160 \%$.

The low-frequency dielectric parameters are more important in reflecting the electrical properties of the extracellular fluids. Extracellular fluid is the direct living environment of cells, whose dielectric properties involves strong research interest, as the low frequency electrode polarization makes measurements incredible. The ability to detect disease using dielectric spectroscopy is based on accurate analysis of dielectric spectroscopy and correct extraction of characteristic parameters. The results of the above experiments confirmed the presence of electrode polarization and its significant negative effect. Therefore, it is necessary to consider the electrode polarization effects to obtain accurate dielectric spectroscopy and characteristic parameters in the clinical application of dielectric spectroscopy, e.g., breast cancer detection [11] and anemia detection [12] using dielectric spectroscopy. Also, depolarization correction method proposed in this paper can eliminate the impact of electrode polarization effects, which can lay the foundation for the study of low frequency dielectric properties in the future.

\section{Acknowledgement}

This work was funded by the National Natural Science Foundation of China (Grant No. 61301246) and the Science and Technology Project of Tianjin (12JCYBJC19300).

\section{References}

[1] X.Z. Dong, The development of the bioelectric impedance technologies, Chinese Journal of Medical Physics 21 (2004), 311-320.

[2] G.H. Markx and C.L. Davey, The dielectric properties of biological cells at radio frequencies: Applications in biotechnology, Enzyme and Microbial Technology 25 (1999), 161-171.

[3] C. Gabriel, A. Peyman and E.H. Grant, Electrical conductivity of issue at frequencies below $1 \mathrm{MHz}$, Physics in Medicine and Biology 54 (2009), 4863-4878.

[4] L. Hermann, Ueber eine wirkung galvanischer ströme auf muskeln und nerven, pflügers archive-The effects of electrical current on muscle and nerve, European Journal of Physiology 6 (1872), 312-360.

[5] B. Rigaud, J.P. Morucci and N. Chauveau, Bioelectrical impedance techniques in medicine, part I: Bioimpedance measurement, second section: Impedance spectrometry, Critical Reviews in Biomedical Engineering 24 (1995), 257-351.

[6] X. Lin, Studies on impedance spectroscopy measurement methods and characteristic of biological tissues, Ph.D. Dissertation, Fourth Military Medical University, 2005.

[7] X.Y. HE and Q. MA, The dielectric of rat blood cells in the frequency range of $0.1 \mathrm{MHz} 100 \mathrm{MHz}$, Chinese Journal of Medical Physics 23 (2006), 268-270.

[8] S.K. Zhao, Dielectric spectroscopy of biological tissues, biological macromolecules solution and related system, in: Dielectric Spectroscopy Methods and Applications, H. LIANG, ed., Chemical Industry Press, Beijing, 2008, pp. 236-262. 
[9] F. Bordi, C. Cametti and T. Gili, Reduction of the contribution of electrode polarization effects in the radio wave dielectric measurements of highly conductive biological cell suspensions, Bioelectric Chemistry 54 (2001), 53-61.

[10] V. Raicu, T. Saibara and A. Irimajiri, Dielectric properties of rat liver in vivo: a noninvasive approach using an open-ended coaxial probe at audio/radio frequencies, Bioelectric Chemistry and Bioenergetics 47 (1998), 325-332.

[11] M.R. Stoneman, M. Kosempa, W.D. Gregory et al., Correction of electrode polarization contributions to the dielectric properties of normal and cancerous breast tissues at audio/radio frequencies, Phys. Med. Biol. 52 (2007), 6589-6604.

[12] L. Chen, Study on dielectric spectroscopy of erythrocytes in suspension from patients with iron deficiency anemia, Mc.S. Dissertation, Ningbo University, 2011. 